\title{
The value of complementary approaches in evolutionary research: reply to Magalhães and Matos
}

\author{
Trends in Ecology and Evolution 27:650-651 (2012)
}

Tadeusz J. Kawecki ${ }^{1}$, Richard E. Lenski ${ }^{2}$, Dieter Ebert ${ }^{3}$, Brian Hollis ${ }^{4}$, Isabelle Olivieri ${ }^{5}$, Michael C. Whitlock $^{6}$

\author{
${ }^{1}$ Department of Ecology and Evolution, University of Lausanne, Switzerland, Email tadeusz.kawecki@unil.ch \\ ${ }^{2}$ BEACON Center for the Study of Evolution in Action, Michigan State University, East Lansing, Michigan 48824, USA, \\ Email lenski@msu.edu \\ ${ }^{3}$ University of Basel, Zoological Institute, Vesalgasse 1, 4051 Basel, Switzerland, Email dieter.ebert@unibas.ch \\ ${ }^{4}$ Department of Ecology and Evolution, University of Lausanne, Switzerland, Email brian.hollis@unil.ch \\ ${ }^{5}$ Université Montpelier 2, CNRS, Institut des Sciences de l'Evolution, UMR 5554, 34095 Montpelier cedex 05, France, \\ Email isabelle.olivieri@univ-montp2.fr \\ ${ }^{6}$ Department of Zoology, University of British Columbia, Vancouver, Canada V6T 1Z4, Email whitlock@zoology.ubc.ca
}

Magalhães and Matos [1] criticize our review of experimental evolution [2] for not discussing the limitations of other research approaches used in evolutionary biology. While we agree that the strengths of experimental evolution result in part from the ability to circumvent some of those limitations, we felt that discussing the limitations of comparative, phylogenetic, paleontological, and other approaches was beyond the scope of our paper. The power of experimental evolution is manifest in the breadth and depth of insights gained through its application, as reviewed in our paper [2]. Nonetheless, experimental evolution does have its own particular limitations as a research approach, and it is important that practitioners of experimental evolution are aware of them to avoid incorrect interpretation of results.

We believe that the "gold standard" for addressing many evolutionary questions is one that uses multiple research approaches and methods that address complementary aspects of the issues at hand. Theory provides a broad framework about what might be possible and links specific assumptions with predictions, thus inspiring empirical tests and sometimes generating unexpected new insights. Experimental evolution can show what outcomes are plausible and accessible in a biological system with particular properties (e.g., population size and mode of reproduction). Studies of phenotypic and genetic variation in natural populations can establish how much and what kinds of variation exist for traits of interest; such studies may also show whether a particular process has occurred in nature (e.g., by detecting signatures of recent selection in the genome). Molecular biology may reveal the mechanisms by which genetic differences give rise to variation in phenotypes and fitness. Finally, phylogenetically-based comparative approaches (and, for traits preserved in the fossil record, paleontology) reveal which of many possible evolutionary scenarios actually occurred, and whether the processes or factors of interest are sufficiently important or general to contribute to broad-scale patterns of differentiation within and among species or higher taxa.

The concerted application of such complementary approaches can synergistically advance our understanding of an evolutionary phenomenon. A case in point is the evolution of aging. Insights by Peter B. Medawar and George C. Williams, later formalized in mathematical models (e.g., [3]), posited that senescence is an expected outcome of evolution (rather than an unavoidable result of attrition) and, moreover, predicted testable connections between aging, reproduction, and extrinsic mortality. Experimental evolution studies verified those predictions under laboratory conditions and demonstrated 
that lifespan can evolve rapidly in either direction (e.g., [4]). Quantitative genetic studies of natural populations confirmed the existence of ample genetic variation in the rate of aging as well as antagonistic pleiotropy between early- and late-life fitness components (e.g., [5]) including in humans (e.g., [6]). Genomic methods are beginning to identify some of the underlying polymorphisms (e.g., [7]), while other high-throughput methods shed light on the architecture of lifespan and related traits (e.g.. [8]). Also, experiments with mutants, transgenics and pharmacological manipulations suggest candidate physiological mechanisms (e.g., insulin signaling) that might modulate the trade-off between early reproduction and lifespan while comparative studies show that these mechanisms are highly conserved across metazoans [9]. Some candidate genes show predicted patterns of geographic variation in allele frequencies across populations (e.g., [10]). Finally, phylogenetically-based comparative studies (e.g., [11]) indicate that the factors identified by theory and in evolution experiments drive much of the variation in lifespan across taxa. Although many questions remain unanswered, the application of complementary approaches has allowed the evolution of aging to become a mature area of research with some potentially important biomedical applications (e.g, [12]).

In conclusion, experimental evolution is a powerful approach for studying evolution based on its particular strengths $[1,2]$ as well as its ability to complement other approaches. The growth of experimental evolution in recent years suggests that this approach was underutilized in the past. We hope and expect that evolutionary biologists will continue to use all available approaches, alone and in concert, to advance our understanding of evolution.

\section{References}

1 Magalhães, S. and Matos, M. (201?) Strengths and weaknesses of experimental evolution: comment on Kawecki et al 2012. Trends Ecol. Evol.

2 Kawecki, T.J., et al. (2012) Experimental evolution. Trends Ecol. Evol. 27, published online

3 Hamilton, W.D. (1966) The moulding of senescence by natural selection. J. Theor. Biol. 12, 12-45

4 Rose, M.R. (1984) Laboratory evolution of postponed senescence in Drosophila melanogaster. Evolution 38, 1004-1010

5 Wilson, A.J., et al. (2007) Evidence for a genetic basis of aging in two wild vertebrate populations. Current Biology 17, 2136-2142

6 Kuningas, M., et al. (2009) Selection for genetic variation inducing pro-inflammatory responses under adverse environmental conditions in a Ghanaian population. PLoS ONE 4, A174-A180

7 Van Bers, N.E.M., et al. (2012) The design and cross-population application of a genome-wide SNP chip for the great tit Parus major. Mol. Ecol. Resour. 12, 753-770

8 Ayroles, J.F., et al. (2009) Systems genetics of complex traits in Drosophila melanogaster. Nat. Genet. 41, 299-307

9 Tatar, M., et al. (2003) The endocrine regulation of aging by insulin-like signals. Science 299, 1346-1351

10 Paaby, A.B., et al. (2010) Identification of a candidate adaptive polymorphism for Drosophila life history by parallel independent clines on two continents. Mol. Ecol. 19, 760-774

11 Blanco, M.A. and Sherman, P.W. (2005) Maximum longevities of chemically protected and nonprotected fishes, reptiles, and amphibians support evolutionary hypotheses of aging. Mechanisms of Ageing and Development 126, 794-803

12 Van Bodegom, D., et al. (2007) Regulation of human life histories the role of the inflammatory host response. In Biogerontology: Mechanisms and Interventions (Rattan, S.I.S. and Akman, S., eds), pp. 8497, Blackwell Publishing 